\title{
PYOPNEUMOTHORAX AND PNEUMOTHORAX
}

\section{A REPORT OF TWO CASES WITH INTERESTING CLINICAL AND ROENTGENOLOGIC FINDINGS*}

\author{
JAMES A. HONEIJ, M.D. \\ NEW HAVEN, CONN.
}

Pneumothorax alone, or accompanied by serous fluid, or by pus, is infrequent enough in any single clinic to be of sufficient interest to study and report. In the present case of pyopneumothorax (Case 1) there is added interest on account of the obscured physical signs and symptoms, the unusual partial encapsulation of the pneumothorax, as shown by roentgenogram, and the transmitted cardiac pulsation through the fluid.

In the majority of cases the collapsed lung is easily discernible by Roentgen ray and can be made out by percussion near the mediastinal border, in contrast to the hyperresonant, tympanitic condition of the rest of the chest, and at the base of the pleural cavity the fluid can be easily marked out. In a typical case of pneumothorax, such as is reported here, and which preceded the present case of pyopneumothorax by a few weeks, diagnosis is not a difficult matter. When atypical complicated cases present themselves, diagnosis is increasingly difficult.

The normal pleura is not visible by roentgenography. When, however, pathologic changes occur, and there is thickening of the pleura, or when definite bands of adhesions result, then such changes are capable of being diagnosed by roentgenographic methods.

If there is serous fluid in the pleural cavity sufficient to give physical signs or symptoms, and if there is with it a pneumothorax condition, then the roentgenoscopic examination will determine it.

In partial pneumothorax conditions, pleural adhesions may, to a certain degree, limit the extent of both the air and fluid in the cavity. If the adhesions are along the median line, the displacement of the heart by the fluid and air is restricted; in cases in which the adhesions occur between diaphragm - especially in its central and dome portion - and pleura or mediastinum, a true comparison of the position of the diaphragm is impossible, and when adhesions extending transversely across the pleural cavity occur - especially in the upper onethird of the chest - the true extent of the pneumothorax and the position and the outline of the diseased lung cannot be determined. Likewise, is it impossible to determine the existence of tuberculosis

* Submitted for publication April 23, 1917. 
in the upper lobe of the lung - if not collapsed - or in any part of the atelectatic lung.

The presence of large amounts of fluid adds to the difficulty of diagnosis, as a diagnosis of fluid in the pleural cavity, as such, is of little value unless the associated condition of the chest contents and their function can be given.

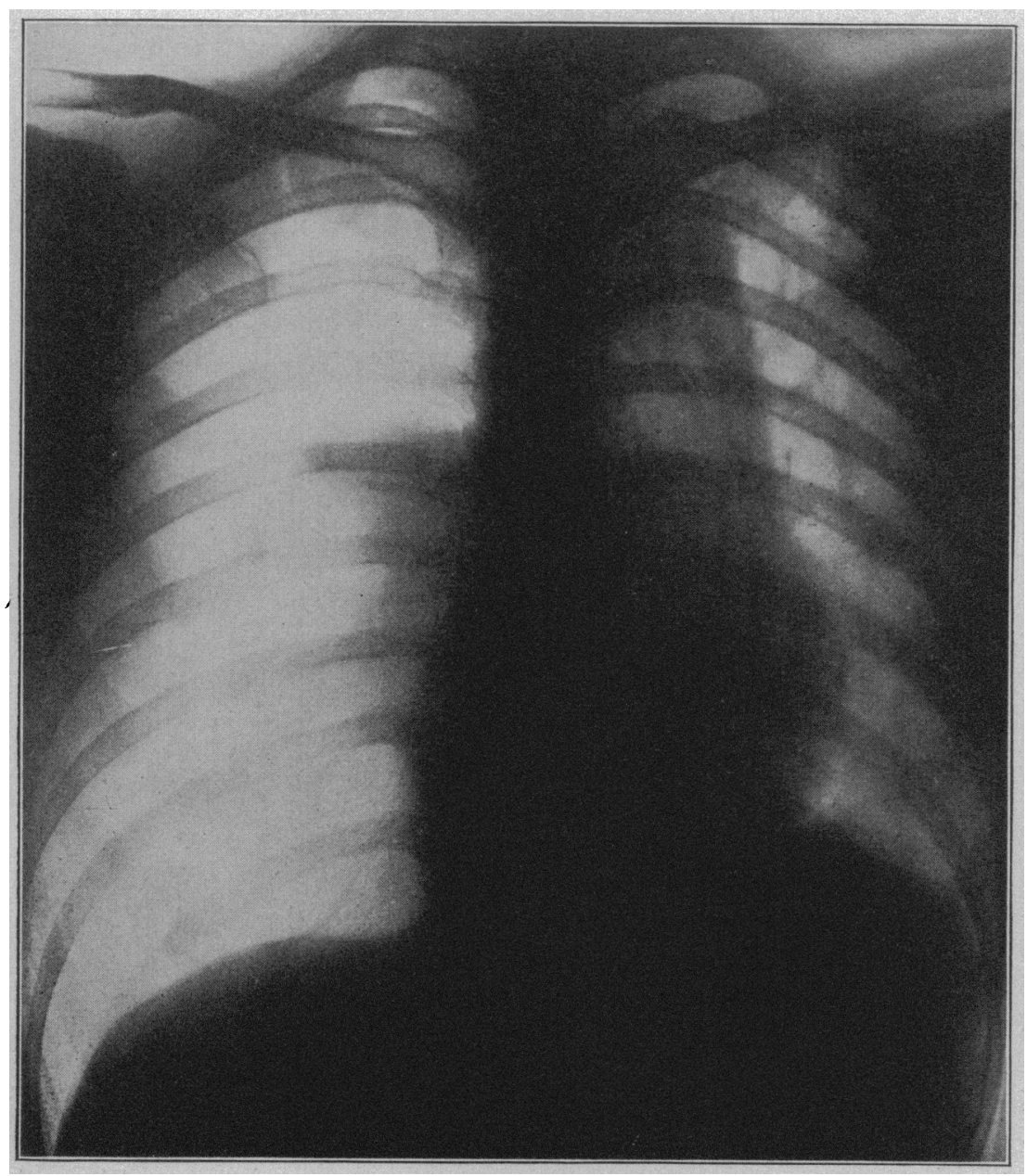

Fig. 1.-Case 1 (Hosp. No. 214). Pneumothorax of left chest; diaphragm at twelfth rib; collapsed lung visible between serenth and tenth ribs, near mediastinum; heart displaced to right.

In probably no other condition of the chest is the value of combined clinical and roentgenographic methods so well illustrated as in the pneumothorax, hydrothorax or pyopneumothorax cases. In Case 1, of pyopneumothorax, this is well brought out. 


\section{REPORT OF CASES}

CASe 1.-(Hosp. No. 214.) Pneumothorax.

History.-March 8, 1917. Woman, aged 27, with a history of a slight attack of vomiting on March 3; no history of pain in the chest; patient has lost 15 pounds in the previous six weeks.

Physical Examination.-The patient is of fairly normal build and development. Physical examination shows a slight bulging of the left chest, with intercostal spaces flush with the ribs; the percussion note is higher pitched on the left and is tympanitic; respiration is faint, and amphoric breathing is heard over the entire left side; definite coin sound elicited. The heart is displaced to the right $10 \mathrm{~cm}$. from the median line.

The roentgenograms show a marked contrast between the right and left lung. The right lung is dense-apparently compressed-as evidenced by the increased shadows at the hilus. Throughout the lung, and especially at the apex, definite mottling is seen. The left chest is transparent-a pneumothorax condition-and near the hilus is the more or less butterfly-wing outline of the atelectatic lung.

The heart and mediastinal vessels are displaced to the right to a considerable extent. The diaphragm on the left is lower than normal; the diaphragm on the right is higher than normal.

Diagnosis.--Pneumothorax, atelectasis. Probable pulmonary tuberculosis. Displaced heart.

March 9, patient aspirated and percussion of the heart shows it to be $7 \mathrm{~cm}$. to the right of the median line; physical signs the same.

Roentgenograms show the heart slightly less displaced by approximately $1 \mathrm{~cm}$. The collapsed lung is denser and the remaining left chest space is slightly less transparent. The costocartilaginous articulations show marked calcification.

On discharge (March 12) breath sounds over left lung still diminished and coin sound distinct; apex beat neither visible nor palpable.

CASE 2.-(Hosp. No. 291.) Hydropneumothorax.

History.-March 29, 1917. Man, aged 51, with history of shortness of breath for five weeks, which has been more marked in the previous three weeks; pain in left chest.

Physical Examination.-The patient is of normal build and fair development, but emaciated. Physical examination shows both apices to be sunken, clavicles prominent; dulness of right apex to the second rib; breathing is harsh and a few râles are heard at the right apex after coughing; tactile fremitus in right axilla; Grocco's sign on the right side; on the left there is dulness from the ninth dorsal spine downward, which is modified when the patient lies on his right side. There is visible and palapable pulsation of the chest in the left post axillary line from the middle of the scapula down to the base; heart displaced to the right.

Diagnosis.-The clinical diagnosis suggested possibly an aneurysm, pericardial effusion and pulmonary tuberculosis.

The patient was partially delirious and difficult to control; was unable to lie down, and suffered with frequent paroxysms of pain in the lower left chest, making the examination difficult both from a clinical and roentgenographic point of view.

The roentgenograms show the heart much displaced to the right. The displacement arises at the first rib and extends gradually and obliquely downward to the right base. The right heart outline is distinctly seen converging at right angles to the diaphragm. The lung on the right is not clear, although it is emphysematous and the diaphragm is very low. The left lung shows marked density and mottling at the apex and to the first space, where it is definitely demarcated by a dense semicircular line $1 \mathrm{~cm}$. wide, which arises from the hilus region and extends upward and outward to the axilla. Below this curved 
line there is a large, clear transparent area, more or less oval in outline, extending to the fifth rib. From the fifth rib downward great density occurs. The diaphragm is not discernible. Barium sulphate in emulsion was given the patient to determine the position of the fundus of the stomach; the roentgenograms show this organ to be situated low in the pelvis, and consequently bears no relation to the process above.

Fluoroscopic examination indicates the surface line of the fluid at the base to be more horizontal than appears on the roentgenograms, and with inspiration there is a slight movement of this line.

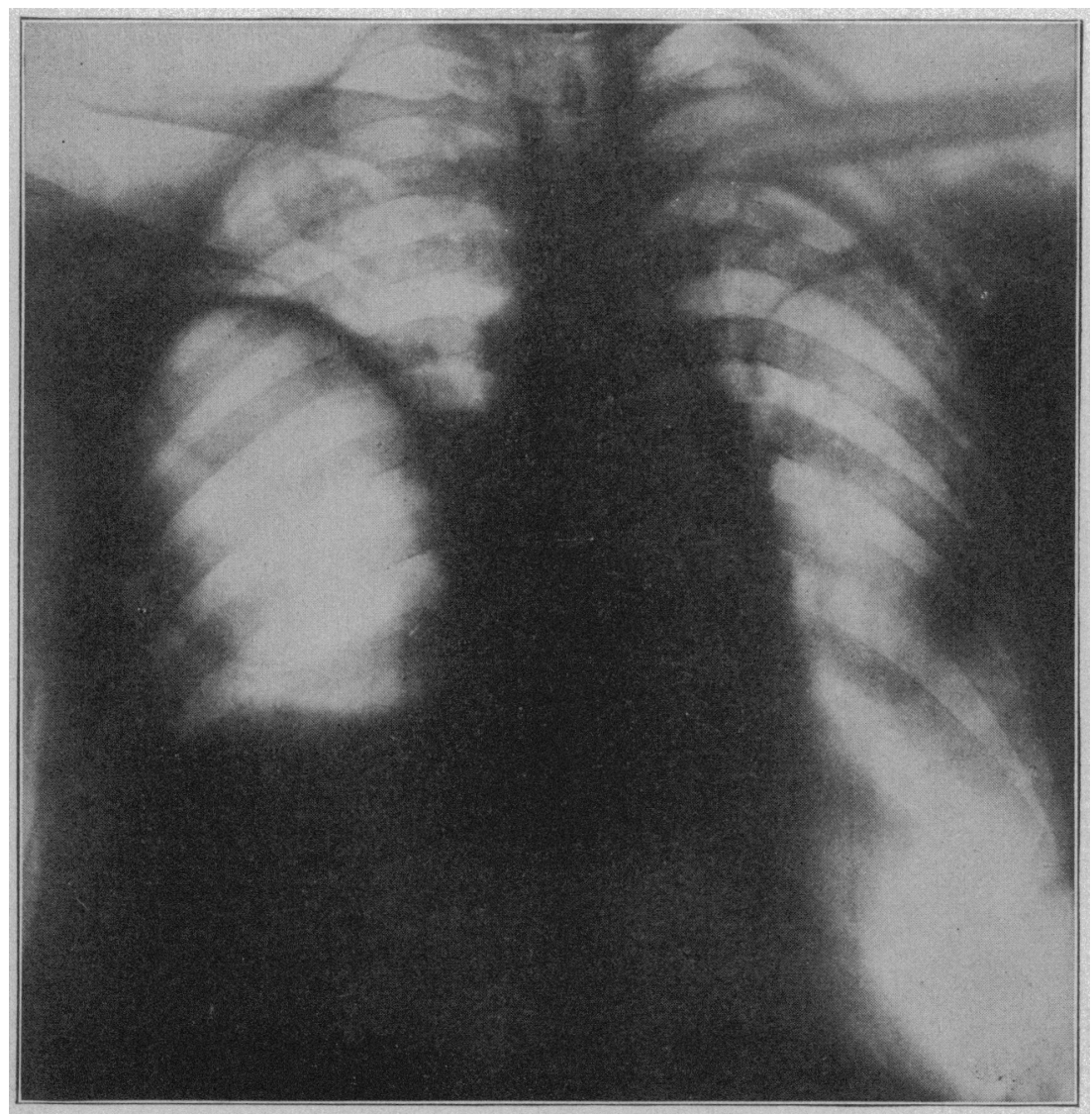

Fig. 2.-Case 2 (Hosp. No. 291). Hydropneumothorax. Patient erect; shows line of fluid on left at ninth space; band of adhesions seen transversely across upper part of chest from seventh rib near mediastinum to middle of scapula on the outer side; compensatory emphysema on the right; diaphragm at eleventh space; heart displaced to right.

Displacement of the heart to the right as shown in this case, without greater symptoms and physical signs, does not suggest aneurysm. The heart outline on the right, especially at its juncture with the diaphragm, suggests some fluid in the pericardium. The density at the left apex and the zone of transparency below this indicates a pnetmothorax-atelectasis of the lower lobe-with fibrous 
adhesions encapsulating the pneumothorax area. The greater density at the base is probably fluid.

The pulsation cannot be explained in any other way than that it is transmitted through the fluid to the outer chest wall from the heart. Were the pulsation present in the upper axilia only, it might be explained by the fibrous adhesions and compressed lung.

April 3. Clinical examination elicits a shifting dulness at the base when the patient shifts his vertical position to the semiprone or horizontal. Above this area a definite coin sound is now obtained; succussion is heard. The roentgenographic diagnosis is verified.

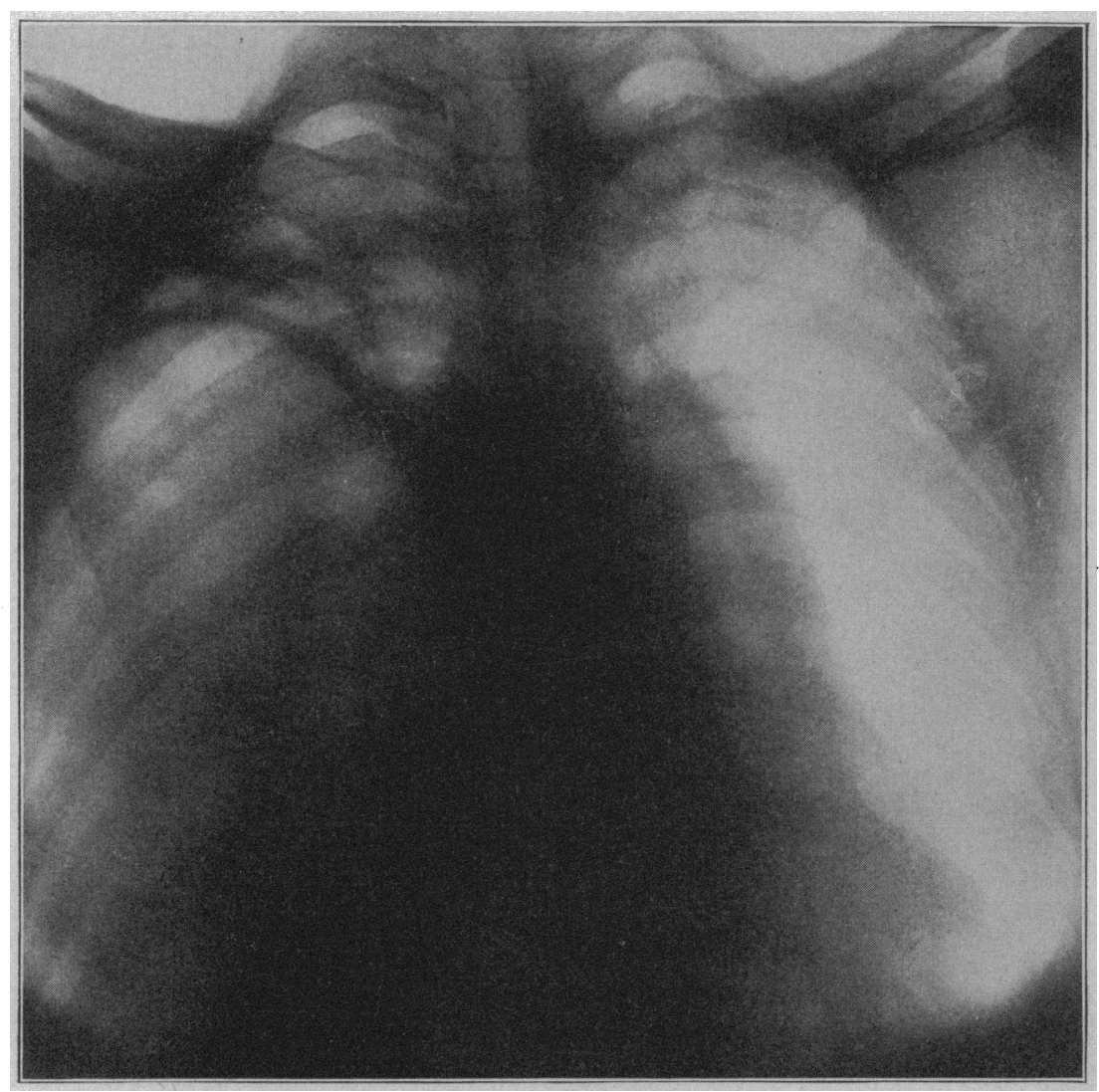

Fig. 3.-Case 2 (Hosp. No. 291). Hydropneumothorax. Patient prone, shows fluid throughout left chest to the curved band of adhesions. For comparison with Figure 2 .

April 5. The patient is aspirated and approximately 11 ounces of foulsmelling pus removed.

April 6. Repeated reentgenographic examination shows the following changes: The density of the left base extends perhaps a little higher, and the density of the upper left lung and the whole of the right show a more detailed mottling than previously seen. With the patient in the prone position the roentgenogram shows that the density seen previously at the left base has extended upward and is now apparent throughout the whole of the left chest, 
even extending beyond the curved zone previously described. This definitely proves the presence of free fluid in the left pleural cavity.

April 7. Resection of ribs. Operative findings: Pleural cavity contains about 1,000 c.c. of very foul, moderately thick pus. The upper lobe of the left lung, as far as could be felt, collapsed, but apparently normal. The lower lobe of the left lung is partially destroyed, the remaining portion being very much infiltrated. The heart is displaced beyond the vertebral column to the right; pericardium much thickened and contains a moderate amount of clear fluid; heart about normal, in size, and there is a dense adhesion between the apex and pericardial side. The patient died on this date.

It is of interest to note that an almost identical case of hydropnenmothorax is reported by Aimé, ${ }^{1}$ occurring in a French soldier infected with pulmonary tuberculosis. The pleural cavity was divided by a fibrous band extending from the diaphragm upward to the compressed lung, forming an outer and an inner air pocket; also dividing the fluid at the base of the lung into two portions. The diagram and roentgenogram are reproduced here.

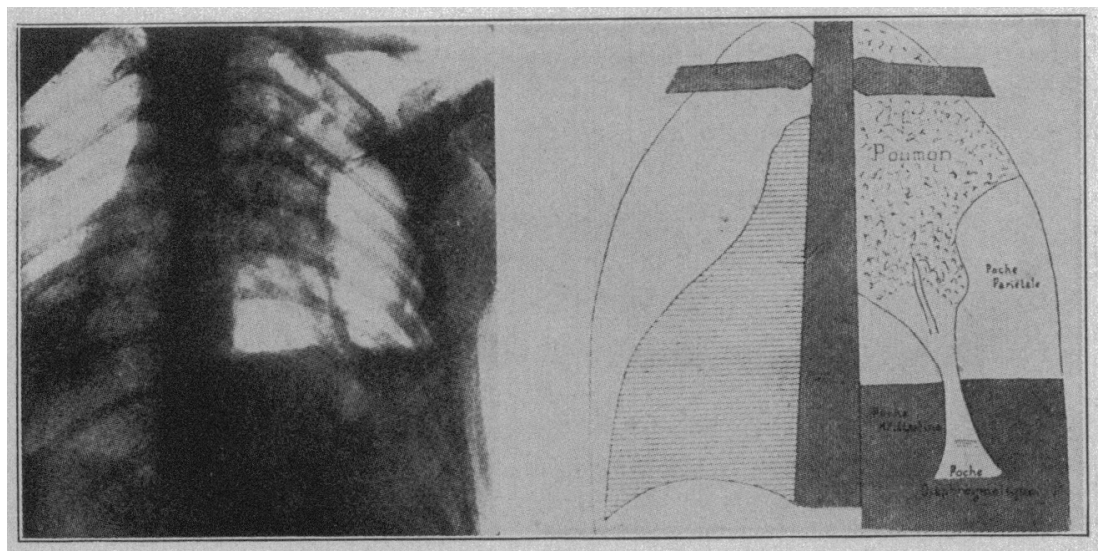

Fig. 4.-On the left, roentgenogram of Aimés case of right hydropneumothorax with pockets. At the right is a tracing of the figure on the left.

In respect to the pulsation described in Case 2 (Hosp. No. 291), a very interesting article is reported by W. P. Herringham. ${ }^{2}$ Among other cases he reports one of pulsating hemopneumothorax in a soldier, as follows :

Hit July 29, 1916, by a bullet on the outside of left shoulder. Hemoptysis followed.

July 30. Heart's apex impalpable; right border a little displaced to the right (by percussion and auscultation). Lungs: right, front, natural; left, front, surgical emphysema obscured everything.

1. Aimé, P.: Right Hydropneumothorax wtih Several Pockets in a Tuberculous Individual, Jour. de radiol. et d'electrol., 1916, 2, 323; abstr., Am. Jour. Roentgenol., 1917, 4, 199.

2. Herringham, W. P.: On the Early Stage of Wounds of the Chest, Quart. Jour. Med., 1916-1917, 10, 79. 
August 5. On the left side an impulse thought to be due to the cardiac apex was felt in the fifth space. The usual cardiac dulness seemed to be present. It was continuous with the impairment of the left side and back. The upper front was tympanitic. There was no breath sound; no bell sound. Pulsation was palapable up to the clavicle.

August 6 . The left side was bulging still and there was a slight local prominence under the clavicle. Pulsation as on August 5, but the heart was obviously beating on the right side as far out as the nipple line. The impulse felt in the left fifth space both August 5 and 6 could not therefore be cardiac. It was the same as the impulse felt in the upper resonant area. An occasional tinkling sound was heard.

August 8. Same signs; well-marked succussion splash both heard and felt; general condition excellent; no distress; temperature normal. Evacuated to base.

The author then concludes by stating that "Pulsating empyema is known, but I have never heard of pulsation in a pleura containing air and blood. When the pulsation was first noticed my colleagues in the clearing station and I held long consultations over the case. We could think of no known condition likely to produce it except aneurysm. But the impulse was not like that of an aneurysm; there was no bruit and an aneurysm of that size must have produced distress. In the absence of any constitutional symptoms pyopneumothorax can be excluded."

From a diagnostic and physical point of view it is obviously of little importance what the medium is through which pulsation takes place, as long as it is fluid. Certainly from the roentgenologist's standpoint it is important.

In both these cases fairly definite signs of pulmonary tuberculosis were present, and, as is well known, tuberculosis is the primary cause of this interesting condition in the majority of cases.

I am indebted to Dr. George Blumer and Dr. Wilder Tileston for these cases, on whose services they occurred. 